http://jmscr.igmpublication.org/home/

ISSN (e)-2347-176x ISSN (p) 2455-0450

crossref DOI: https://dx.doi.org/10.18535/jmscr/v7i7.100

\title{
Effect of Three Plant Extracts and Atorvastatin on Serum Lipids and Lipoproteins in Rats Fed High Cholesterol Diet
}

\author{
Authors \\ Kislay Awasthi ${ }^{1}$, R.C. Gupta ${ }^{2}$, Abbas Ali Mahdi ${ }^{3}$ \\ ${ }^{1}$ National Institute of Medical Sciences, Jaipur-303121 (Raj) \\ ${ }^{2}$ Prof \& Head, Dept. of Biochemistry, National Institute of Medical Sciences, Jaipur (Raj) \\ ${ }^{3}$ Prof \& Vice Chancellor, Era University, Lucknow (U.P.)
}

\begin{abstract}
Introduction: To evaluate the relative efficacy of Gloriosa superba, Valeriana wallichii and Odenlandia corymbosa extracts and atorvastatin in correcting the dyslipidemia induced by feeding high cholesterol diet to rats.

Methods: The study was carried out on healthy male adult rats of Wistar strain. Dyslipidemia was induced by feeding them fructose-rich high fat diet (F-HFD). Gloriosa superba extract, Valeriana wallichii extract, Odenlandia corymbosa extract and atorvastatin were given orally for 30 days along with F-HFD to four different groups. Serum lipids and lipoproteins were measured.

Results: Feeding F-HFD for 30 days led to dyslipidemia in the form of raised serum cholesterol, triglycerides, $L D L-$ cholesterol, VLDL-cholesterol and free fatty acids. In the rats fed F-HFD + Valeriana wallichii extract, serum cholesterol, triglycerides and VLDL-cholesterol were significantly lower as compared to rats fed F-HFD alone. No such effect was seen in the rats given F-HFD + Gloriosa superba extract and F-HFD + Oldenlandia corymbosa extract. In the rats fed F-HFD + atorvastatin, serum lipids were significantly lower as compared to rats fed F-HFD alone and the effect was stronger than that of Valeriana wallichii.

Conclusion: Valeriana wallichii possesses anti-dyslipidemic activity but is not as effective as atorvastatin.

Keywords: Anti-dyslipidemic, Gloriosa superba, Valeriana wallichii, Odenlandia corymbosa.
\end{abstract}

\section{Introduction}

Dyslipidemia is an abnormality of plasma lipids in which plasma total cholesterol (TC), low-density lipoprotein cholesterol (LDL-C), very low-density lipoprotein cholesterol (VLDL-C) and triglycerides (TG) are elevated and high-density lipoprotein cholesterol (HDL-C) is lowered, either singly or in combination ${ }^{1}$. Detection and correction of this condition is important for prevention and control of cardiovascular disease (CVD) and its sequelae. In $80 \%$ cases, lipid abnormalities are related to diet and lifestyle while heredity plays a role in the remaining cases ${ }^{2}$. The prevalence of dyslipidemia is increasing worldwide ${ }^{3,4}$. Studies have shown increased prevalence of dyslipidemia and have attributed it to increasing prevalence of obesity and type 2 diabetes mellitus, which are linked to changes in lifestyle associated with modernization and socioeconomic development ${ }^{5}$.

A number of anti-dyslipidemic drugs are available in modern medicine to treat abnormal plasma lipids. The drugs used for controlling dyslipidemia include fibrates, statins and bile acid-binding resins. The statins are the most widely used out of these. However, long-term use of these drugs is not free from undesirable effects.

Atorvastatin, a widely used lipid-lowering agent, is an inhibitor of HMG-CoA reductase, which catalyzes the conversion of HMG-CoA to 
mevalonate. Inhibition of HMG-CoA reductase decreases cholesterol synthesis and leads to upregulation of LDL cholesterol (LDL-C) receptors in the liver, mediated by activation of sterol regulatory element binding protein resulting in enhanced clearance of LDL from the circulation, thus playing an important role in preventing atherosclerosis ${ }^{6}$.

Some indigenous plants are supposed to possess cardio-protective properties e.g. Gloriosa superba, Valeriana wallichii and Odenlandia corymbosa. Gloriosa superba is commonly known as Glory Lily (Bachnag in Hindi) and belongs to family Liliaceae. It is found commonly in forest and is under cultivation in fairly large areas of India. Valeriana wallichii belongs to Valerianeaceae family and grows in temperate Himalayas from Kashmir to Bhutan. Ithas been used in the Ayurvedic system of medicine for centuries. Its native names include Tagara, Nata, Vakra etc. Odenlandia corymbosa belongs to family Rubiaceae and is commonly known as Diamond Flower (Daman Pappar in Hindi). It is found all over India ${ }^{7}$.

Since plant products are generally safe, the present study was undertaken to evaluate the effectiveness of Gloriosa superba, Valeriana wallichii and Odenlandia corymbosa extracts in correcting the dyslipidaemia resulting from feeding fructose-rich high fat diet to rats and to compare their effect with that of atorvastatin.

\section{Material and Methods}

Authenticated samples of Gloriosa superba, Valeriana wallichii and Odenlandia corymbosa were procured. The plant samples were dried under shade. They were ground by an electric blender, and the resulting powder (200 g) was extracted with one liter of $70 \%$ alcohol for 72 hours at room temperature. The extract was filtered and the solvent was evaporated to dryness. The yield of extract was calculated and stored at $2-8^{\circ} \mathrm{C}$ for further use.

The study was carried out on healthy male adult rats of Wistar strain (200-225 g) obtained from the
Animal House of King George's Medical University, Lucknow (U.P.). The study was started after obtaining permission from the Animal Ethics Committee of the University. All the rats were initially maintained on Hindustan Lever Food Pellets diet and water ad libitium. The cages were kept in a temperature and humidity controlled room with a 12-hour light-dark cycle. The rats were divided into six groups of six rats each and were fed as follows for 30 days:

Group 1- Control rats fed normal pellet diet.

Group 2- Rats fed fructose-rich high fat diet (FHFD).

Group 3- Rats fed F-HFD and given extract of Gloriosa superba $(250 \mathrm{mg} / \mathrm{kg}$ of body weight orally once a day).

Group 4 - Rats fed F-HFD and given extract of Valeriana wallichii $(250 \mathrm{mg} / \mathrm{kg}$ of body weightorally once a day).

Group 5- Rats fed F-HFD and given extract of Odenlandia corymbosa $(250 \mathrm{mg} / \mathrm{kg}$ of body weightorally once a day).

Group 6- Rats fed F-HFD and given atorvastatin $(10 \mathrm{mg} / \mathrm{kg}$ of body weight orally once a day).

After 30 days, serum total cholesterol (TC), triglycerides (TG) and high density lipoproteincholesterol (HDL-C) were quantified by CHODPAP, GPO-POD and PEG-PAP methods respectively, and low density lipoprotein cholesterol (LDL-C) by immuno-turbidimetric assay $^{8}$. Very low density lipoprotein cholesterol (VLDL-C) was calculated by Friedewald's formula ${ }^{9}$. Serum free fatty acids (FFA) were measured by the method of Monsinger et al ${ }^{10}$ and phospholipids (PL) by malachite green method ${ }^{11}$. Plasmalecithin cholesterol acyl transferase (LCAT) activity was measured according to Albers et al ${ }^{12}$ andpost heparin lipolytic activity (PHLA) according to Wind and Robinson ${ }^{13}$.

\section{Statistical Analysis}

Student's unpaired t-test was used for comparison. The $\mathrm{p}$ value $<0.05$ was considered significant. Analysis was carried out on SPSS 16.0 version. 


\section{Results}

The rats fed F-HFD for 30 days had significantly higher serum TC, TG, LDL-C, VLDL-C, HDL-C and FFA than the rats fed normal pellet diet. No significant difference was seen in serum PL, LCAT and PHLA (Table 1). Thus, feeding F-HFD for 30 days produced significant dyslipidemia.

Table 1: Final levels of serum total cholesterol (TC), triglycerides (TG), high density lipoprotein cholesterol (HDL-C), low density lipoprotein cholesterol (LDL-C), very low density lipoprotein cholesterol (VLDL-C), free fatty acids (FFA), phospholipids (PL), lecithin cholesterol acyl transferase (LCAT) and post heparin lipolytic activity (PHLA) in rats fed normal diet and rats fed fructose-rich high fat diet (F-HFD) for 30 days (all the values are mean $\pm \mathrm{SD}$ ).

\begin{tabular}{|c|c|c|}
\hline & Rats on normal diet & $\begin{array}{c}\text { Rats on F- } \\
\text { HFD }\end{array}$ \\
\hline TC $(\mathrm{mg} / \mathrm{dL})$ & $62.6 \pm 9.72$ & $128 \pm 18.2^{* *}$ \\
\hline TG $(\mathrm{mg} / \mathrm{dL})$ & $48.1 \pm 11.2$ & $110 \pm 16.6^{* *}$ \\
\hline HDL-C $(\mathrm{mg} / \mathrm{dL})$ & $28.3 \pm 8.61$ & $40.5 \pm 6.86^{*}$ \\
\hline LDL-C $(\mathrm{mg} / \mathrm{dL})$ & $24.6 \pm 8.2$ & $65.5 \pm 19.2^{* *}$ \\
\hline VLDL-C(mg/dL) & $9.6 \pm 2.2$ & $22 \pm 3.3^{* *}$ \\
\hline FFA $(\mathrm{mg} / \mathrm{dL})$ & $62.5 \pm 18.9$ & $90.1 \pm 11.6^{*}$ \\
\hline PL $(\mathrm{mg} / \mathrm{dL})$ & $102.3 \pm 23.7$ & $128.1 \pm 26.3^{\#}$ \\
\hline LCAT $(\mathrm{nmol} / \mathrm{mL} / \mathrm{h})$ & $36.3 \pm 14.7$ & $44.1 \pm 13.4^{\#}$ \\
\hline PHLA $(\mathrm{nmol} / \mathrm{mL} / \mathrm{h})$ & $21.0 \pm 3.8$ & $21.6 \pm 5.50^{\#}$ \\
\hline
\end{tabular}

${ }^{*} \mathrm{p}<0.05,{ }^{* *} \mathrm{p}<0.001$ and ${ }^{\#} \mathrm{p}>0.05$ as compared to normal diet group
The serum levels of TC,TG,HDL-C, LDL-C, VLDL-C, FFA, PL, LCAT and PHLA of rats given F-HFD + Gloriosa superba extract, F-HFD + Valeriana wallichii extract, F-HFD + Oldenlandia corymbosa extract, andF-HFD + atorvastatin for 30 days were compared with those of rats fed F-HFD alone. No significant difference was seen in the groups fed F-HFD + Gloriosa superba and F-HFD + Oldenlandia corymbosa, and the group fed F-HFD alone. Thus, these two plant extracts had no effect on the serum lipid levels. In the rats fed F-HFD + Valeriana wallichii extract, serum TC, TG, and VLDL-C were significantly lower as compared to the rats fed F-HFD alone. In the rats fed F-HFD + atorvastatin, serum TC, TG, LDL-C, VLDL-C, HDL-C, FFA and PL were significantly lower as compared to rats fed F-HFD alone. Thus both Valeriana wallichii and atorvastatin showed antidyslipidemic effect but the effect of atorvastatin was stronger than that of Valeriana wallichii (Table 2).

Table 2: Final levels of serum total cholesterol (TC), triglycerides (TG), high density lipoprotein cholesterol (HDL-C), low density lipoprotein cholesterol (LDL-C), very low density lipoprotein cholesterol (VLDL-C), free fatty acids (FFA), phospholipids (PL), lecithin cholesterol acyl transferase (LCAT) and post heparin lipolytic activity (PHLA) in rats fedfructose-rich high fat diet (F-HFD), F-HFD + G.superba extract (250 $\mathrm{mg} / \mathrm{kg} \mathrm{BW/day),} \mathrm{F-HFD} \mathrm{+} \mathrm{V.} \mathrm{wallichii} \mathrm{extract}(250 \mathrm{mg} / \mathrm{kg}$ BW/day),F-HFD+ $O$. corymbosa extract (250 $\mathrm{mg} / \mathrm{kg} \mathrm{BW} /$ day)and F-HFD + atorvastatin $(10 \mathrm{mg} / \mathrm{kg}$ BW/day)for 30 days (all the values are mean $\pm \mathrm{SD}$ ).

\begin{tabular}{|c|c|c|c|c|c|}
\hline & F-HFD & $\begin{array}{c}\text { F-HFD }+G . \\
\text { superba } \text { extract }\end{array}$ & $\begin{array}{c}\text { F-HFD }+V . \\
\text { wallichii } \text { extract }\end{array}$ & $\begin{array}{c}\text { F-HFD }+ \text { O. } \\
\text { corymbosa extract }\end{array}$ & $\begin{array}{c}\text { F-HFD }+ \\
\text { atorvastatin }\end{array}$ \\
\hline TC $(\mathrm{mg} / \mathrm{dL})$ & $128 \pm 18.2$ & $120 \pm 16.6^{\#}$ & $91.5 \pm 14.6^{* *}$ & $124.6 \pm 17.0^{\#}$ & $68.3 \pm 11.6^{* * *}$ \\
\hline TG $(\mathrm{mg} / \mathrm{dL})$ & $110 \pm 16.6$ & $90 \pm 11.8^{\#}$ & $70.5 \pm 12.1^{* * *}$ & $99.1 \pm 12.8^{\#}$ & $57 \pm 4.73^{* * *}$ \\
\hline HDL-C $(\mathrm{mg} / \mathrm{dL})$ & $40.5 \pm 6.86$ & $31.6 \pm 5.00^{\#}$ & $30.1 \pm 6.61^{\#}$ & $38.1 \pm 2.85^{\#}$ & $29.1 \pm 6.79^{*}$ \\
\hline LDL-C (mg/dL) & $65.5 \pm 19.2$ & $71.8 \pm 14.9^{\#}$ & $47.2 \pm 11.2^{\#}$ & $70 \pm 17.6^{\#}$ & $27.7 \pm 12.3^{* * *}$ \\
\hline VLDL-C(mg/dL) & $22.0 \pm 3.3$ & $18 . \pm 2.3^{\#}$ & $14.1 \pm 2.4^{* * *}$ & $19.8 \pm 2.5^{\#}$ & $11.4 \pm 0.94^{* * *}$ \\
\hline FFA (mg/dL) & $90.1 \pm 11.6$ & $84.1 \pm 12.4^{\#}$ & $71.5 \pm 11.0^{\#}$ & $86.6 \pm 11.8^{\#}$ & $59.1 \pm 17.1^{*}$ \\
\hline PL (mg/dL) & $128.1 \pm 26.3$ & $102.6 \pm 17.8^{\#}$ & $92.1 \pm 15.7^{\#}$ & $120 \pm 27.1^{\#}$ & $80.6 \pm 3.14^{* *}$ \\
\hline LCAT (nmol/mL/h) & $44.1 \pm 13.4$ & $50 \pm 12.9^{\#}$ & $54.1 \pm 11.5^{\#}$ & $49.1 \pm 9.96^{\#}$ & $59.8 \pm 10.4^{\#}$ \\
\hline PHLA $(\mathrm{nmol} / \mathrm{mL} / \mathrm{h})$ & $21.6 \pm 5.50$ & $16.8 \pm 5.07^{\#}$ & $13.6 \pm 3.14^{*}$ & $17.8 \pm 4.16^{\#}$ & $11.8 \pm 2.63^{*}$ \\
\hline
\end{tabular}

$* \mathrm{p}<0.05, * * \mathrm{p}<0.01, * * * \mathrm{p}<0.001$ and ${ }^{\#} \mathrm{p}>0.05$ as compared toF-FHD group

\section{Discussion}

Dyslipidemia in the form of elevated total cholesterol, low density lipoprotein (LDL) cholesterol, very low density lipoprotein (VLDL) cholesterol and triglycerides and decreased high density lipoprotein (HDL) cholesterol is one of the 
major coronary risk factors. There is a strong pathophysiological association between raised LDL cholesterol level and initiation and progression of coronary atherosclerosis ${ }^{14}$.

Currently available therapies for dyslipidemia in modern medicine, e.g. fibrates, statins and bile acid sequestrates, correct lipid abnormalities to a significant extent but can also produce adverse effects. Therefore, there is a need to develop safe and effective treatment modalities for dyslipidemia. Medicinal plants can provide drugs that are less toxic, have fewer side effects and are cost effective. Some plants are supposed to have cardio-protective effects. If we can find plant products with lipid-lowering effect, they can provide a new option for the treatment of dyslipidemia.

We explored three plants viz. Gloriosa superba, Valeriana wallichii and Oldenlandia corymbosa for their possible anti-dyslipidemic effect in rats. For comparison, we took a standard drug i.e. atorvastatin. Gloriosa superba and Oldenlandia corymbosa did not show an anti-dyslipidemic effect but Valeriana wallichii was found to have a significant anti-dyslipidemic effect. When Valeriana wallichii extract and atorvastatin were compared, it was seen that the effect of atorvastatin was stronger. This finding is in agreement with the report of Zanguei and Jowhary who had investigated the effect of a different species of the same genus i.e. Valeriana officinalis $^{15}$.

The results of the present study show that Valeriana wallichii extract can favorably modify the dyslipidemia resulting from feeding high cholesterol diet to rats. The extract was somewhat less effective than atorvastatin but if we can isolate the active compound from the plant, it may turn out to be more effective. One of the limitations of the present study was small sample size. Studies on larger samples are warranted for robust results.

\section{Conclusion}

Valeriana wallichii extract lowers serum lipid levels but is not as effective as atorvastatin.

\section{References}

1. Bamba V, Rader D. Obesity and Atherogenic Dyslipidemia. Gastroenterology 2007;132(6):2180-90.

2. Eaton CB. Hyperlipidemia. Prim Care Clin Office Pract 2005; 32:1027-55.

3. Tailor AM, Peeters PH, Norat T, Vineis P, Romaguera D. An update on the prevalence of the metabolic syndrome in children and adolescents.cInt $\mathbf{J}$ PediatrObes 2010;5(3):202-13

4. Joshi SR, Anjana RM, Pradeepa R et al. Prevalence of dyslipidemia in urban and ru ral India: the ICMR INDIA study. PLoS One 2014;9 (5).

5. Mula-Abed WS, Chilmeran SK. Prevalence of Dyslipidemia in the Iraqi Adult Population. Saudi Medical Journal 2007;28(12):1855-7.

6. Delsing DJ, Jukema JW, van de Wiel MA et al. Differential effects of amlodipine and atorvastatin treatment and their combination on atherosclerosis in Apo $\mathrm{E}^{*}$ 3-Leiden transgenic mice. $\mathrm{J}$ CardiovascPharmacol 2003;42(1): 63-70.

7. Tripathi I, Nighuntu R. $4^{\text {th }}$ ed. Varanasi: Chaukhambha Krishnadas Academy; 2006.pp.41-42.

8. Hirano T, Ito Y, Saegusa H, Yoshino G. A novel and simple method for quantification of small, dense LDL. J Lipid Res. 2003; 44(11):2193-2201.

9. Friedewald WT, Levy RI, Fredrickson DS.Estimation of the concentration of lowdensity lipoprotein cholesterol in plasma, without use of the preparative ultracentrifuge. Clin Chem. 1972; 18(6):499-502.

10. Mosinger F. Photometric adaptation of Dole's microdetermination of free fatty acids. J Lipid Res. 1965;6(1):157-159. 
11. Chan KM, Delfert D, Junger KD. A direct colorimetric assay for $\mathrm{Ca}^{2+\mathrm{v}}$ stimulated ATPase activity.Anal Biochem. 1986;157(2):375-380.

12. Albers JJ, Chen CH, Adolphson JL. Lecithin: cholesterol acyltransferase (LCAT) mass; its relationship to LCAT activity and cholesterol esterification rate. Journal of Lipid Research. 1981;22(8):1206-13.

13. Wing DR, Robinson DS.Clearing-factor lipase in adipose tissue.A possible role of adenosine 3', 5'-(cyclic)-monophosphate in the regulation of its activity.Biochemical Journal. 1968;109(5):841-9.

14. RaoRS, MisraA, Sharma SK. Recent trends in epidemiology of dyslipidemias in India. Indian Heart Journal 2017; 69: 382392.

15. Zanguei Z, Jowhary H. Hydroalcoholic Extract of Valerian, Blood Factors, and Atherosclerotic Plaque Formation in Male Hypercholesterolemic Rabbits. Biomed Pharmacol J 2015;8(2). 